\title{
Electron acceleration by coherent laser pulse echelons in periodic plasma structures
}

\author{
A. Pukhov ${ }^{1,2}$, I. Kostyukov ${ }^{2,3}$, T. Tückmantel ${ }^{1}$, Ph. Luu-Thanh ${ }^{1}$ \\ ${ }^{1}$ University of Dusseldorf, 40025 Germany \\ ${ }^{2}$ University of Nizhny Novgorod, Nizhny Novgorod 603950, Russia and \\ ${ }^{3}$ Institute of Applied Physics RAS, Nizhny Novgorod 603950, Russia
}

\begin{abstract}
We consider a possibilty to use an echelon of mutually coherent laser pulses generated by the emerging CAN (Coherent Amplification Network) technology for direct particle acceleration in periodic plasma structures. The plasma structure survives a single shot only. However, due to it's simplicity and projected very low production costs, the structure can be replaced for every laser shot at a kiloherz repetition rate. We discuss resonant and free streaming configurations. The resonant plasma structures can trap energy of longer laser pulses but are limited to moderate laser intensities of about $10^{14} \mathrm{~W} / \mathrm{cm}^{2}$ and are very sensitive to the structure quality. The free streaming configurations can survive laser intensities above $10^{18} \mathrm{~W} / \mathrm{cm}^{2}$ for several tens of femtoseconds so that sustained accelerating rates well above $\mathrm{TeV} / \mathrm{m}$ are feasible. In our full electromagnetic relativistic particle-in-cell (PIC) simulations we show a test electron bunch gaining up to $120 \mathrm{GeV}$ over a distance of $5.3 \mathrm{~cm}$ only.
\end{abstract}




\section{INTRODUCTION}

More than twenty years ago the invention of chirped-pulse-amplification (CPA) technique triggered a revolution in laser technology and lead to ultrashort laser pulses with huge peak powers [1]. The novel laser sources allowed for a steady advance in a number of important scientific and industrial applications: high-field physics, "attosecond" physics and high-order harmonic generation, secondary compact sources of particles and radiation, inertial confinement fusion, probing and imaging of ultrafast processes for biology, medicine and material sciences, etc. The impressive progress has been demonstrated in plasma-based acceleration methods with ultrashort high-power laser pulses used as a driver generating accelerating plasma structures. Quasimonoenergetic electron bunches with the energy up to $1 \mathrm{GeV}$ have been generated in the pioneering experiments [2 [5]. Now the $1 \mathrm{GeV}$ energy barrier has been broken and electron bunches with a few percent energy spread and unprecedented submilliradian divergences have been recorded at $2 \mathrm{GeV}$ energy [6]. In these experiments, the electrons have been accelerated in underdense plasmas in the so called bubble regime of laser wake fields [7]. The traditional CPA lasers use TiSa crystals and deliver an average power of a few Watts only and a low efficiency. This is a significant drawback when we think about high energy physics applications where high luminosities are usually required.

Another laser technique based on fiber amplifiers pushes the laser average power up to kilowatt level and the laser wall-plug efficiency to over 30\%. These values are several orders of magnitude better than the traditional TiSa lasers could possibly provide [8, 9]. The high peak power required by many applications can be achieved when the fiber laser systems are combined with CPA pulse compression and massive parallelism of multiple fiber amplifiers [10, 11].

The real revolution however is promised by the CAN (Coherent Amplification Network) technology [12]. Here, the high average power and efficiency of the fiber laser systems is accomplished by the mutual coherence of individual laser pulses [13] in the massively parallel fiber array. For the first time we are going to have a huge number of identical - and thus mutually coherent - laser pulses that can be flexibly arranged. It is expected that the laserdriven particle acceleration schemes will strongly benefit from the CAN project [12]. The coherency allows to control not only the space-time distribution of the laser intensity in the acceleration region but also the phase distribution. This promises to make the acceleration 
much more robust and efficient.

At the same time, the continuous progress in micro- and nanotechnology stimulates exploration of dielectric-based laser-driven acceleration structures [14]. Such structures transform the transverse laser field into accelerating and focusing fields suitable for efficient particle acceleration. Various accelerating schemes have been suggested: traveling wave structures [15], semi-open structures [16], resonant-closed structures [17], photon crystal structures [18],

phase-modulation-mask structures [19] etc. However, all these structures were designed for a single laser pulse driver as the traditional laser technology provides coherence for a single laser pulse only.

Here we show that the CAN technology may become a real game changer in the structured acceleration. We propose several schemes that combine the advantage of multiple coherent laser pulses with advantages of resonant-closed and phase-modulation-mask structures. We concentrate here on single-shot regime when the solid elements of the structure are converted to plasma by the field ionization and evaporate after the interaction. Yet we assume that the structure keeps its integrity during the short laser pulse duration. Thus, we generate a regular overdense plasma structure. In contrast to the previous dielectric-based schemes [17, 19] we analyze robust schemes which do not rely on the laser phase shift in solid elements of the structure because this would be difficult to control in plasma.

The regular plasma structures allow for the first time to use the huge laser field itself and not a small perturbation generated by the laser in plasma. The sustained accelerating field can thus reach unprecedented values up to several $\mathrm{TV} / \mathrm{m}$.

\section{ACCELERATING STRUCTURE BASED ON HALF-PERIOD FABRY-PEROT PLASMA RESONATORS}

First we propose an accelerating structure based on a periodic set of Fabry-Perot resonators. The structure consists of two-dimensional resonator boxes on top of a solid substrate. The both sides of the cavities are equal to half a laser wavelength. The cross section of the structure geometry is shown in Fig 1 . The cavities are covered by a thin foil (the dashed area in Fig. 1) that is partially transparent for laser radiation while the other cavity walls reflect radiation. The microcavities (the white quadratic areas in Fig. 1b) may provide field enhancement up to the factor 


$$
\frac{E_{\mathrm{c}}}{E_{0}} \approx \frac{1}{1-R}
$$

where $E_{c}$ is the field in the cavity, $E_{0}$ is the laser field, $R$ is reflectivity of the covering foil. It has been demonstrated previously [21] that intensity in the plasma cavity may reach a 100-fold that of the pump lasers.

The field amplification (1) is important for laser pulses that are several periods long. The pulse energy gets trapped in the resonator and can be harvested by the witness beam load. The pulse duration $\tau_{p}$ and the optimal reflectivity of the semitransparent foil are connected as

$$
\tau_{p} \approx \frac{\pi \lambda}{2 c(1-R)}
$$

where $\lambda$ is the laser wavelength.

To achieve a phase synchronism between accelerated particles with the velocity $v$ and the field, the microcavities must be periodically intermitted along the beam axis with the period

$$
\Lambda=\lambda \frac{v}{c}
$$

Thus, we introduce overdense plasma hills of $\lambda / 2$ width and $\lambda$ period to screen the laser field in its decelerating phase (the grey parts in Fig 1). The screening plasma hills should have a hole along the structure axis for particle beam transportation. The structure is irradiated from the $+x$ direction by a laser polarized in the $y$ direction. The relativistic electron moves along the $y$ axis through the resonator cavity and through the screening elment within the hole of radius $r$. An infinite extent in the $z$ dimension is assumed.

The laser pulse must be short enough and the laser intensity must be moderate so that the structure keeps its integrity during the interaction. Our PIC simulations using the code VLPL (Virtual Laser Plasma Lab) 20] show that the plasma resonator structures may survive intensities up to about $10^{14} \mathrm{~W} / \mathrm{cm}^{2}$, corresponding to accelerating fields above $10 \mathrm{GV} / \mathrm{m}$.

The principle of the proposed accelerating structure is rather simple: enhancement of the accelerating fields and damping of the decelerating fields. The relativistic electron with a 


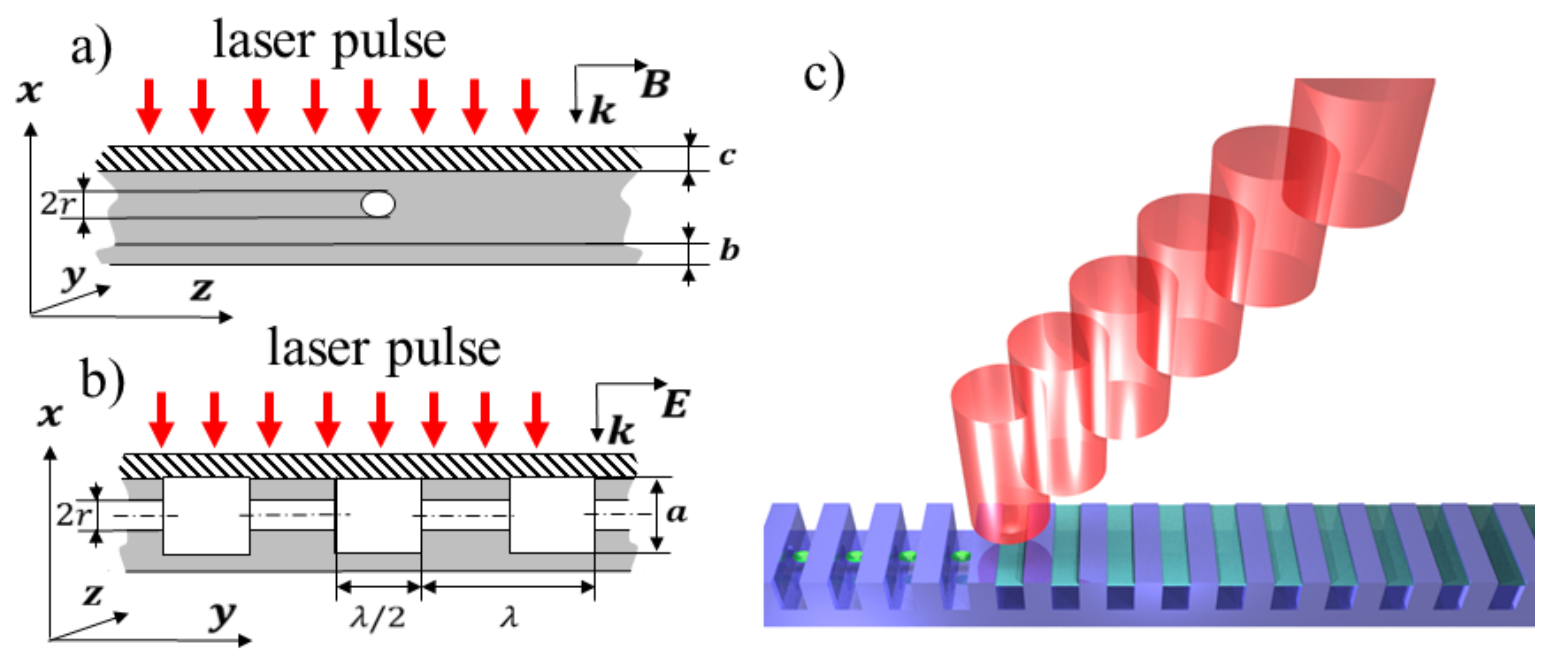

Figure 1: Schematic plot of the resonator accelerating structure: a) cut across the witness beam axis; b) cut along the witness electron beam axis; c) three dimensional view of the periodic resonator structure. Individual laser pulses in the echelon are phased to each other and arrive at the structure with a delay corresponding to the particle travelling time. The dashed area in the two dimensional cuts is the semi-transparent thin foil; the grey area is the overdense plasma elements; the white areas are vacuum microcavities and the microchannel for the particle beam transport.

proper phase harvests the accelerating field in the cavity during the half laser period. When the laser field changes its sign and becomes decelerating, electrons are screened from the field under the overdense plasma hill. Relativistic electrons passing through the structure at a proper phase gain energy.

In our PIC simulations, we took a laser pulse with a Gaussian temporal profile $a(t)=$ $a_{0} \exp \left(-t^{2} / 2 \tau_{p}^{2}\right)$ with the relativistic amplitude $a_{0}=e E_{L} /\left(m c \omega_{L}\right)=0.01$ and duration $\tau_{p}=10 \lambda / c$. Here $\lambda=800 \mathrm{~nm}$ is the laser wavelength, $E_{L}$ is the laser field amplitude, $\omega_{L}$ is the laser frequency, $m, e$ and $c$ are the electron mass, the electron charge and the speed of light, respectively. The plasma electron density was assumed to be $n_{e}=4 \times 10^{23} \mathrm{~cm}^{-3}$, the thickness of the semitransparent foil was $d=0.02 \lambda$. The longitudinal electric field distribution calculated in the cavity and in the damping part is shown in Fig 2 a. It is seen from Fig 2 a that the electric field is amplified by a factor about 3 in the cavity due to the Fabry-Perot effect while it is supressed in the damping part nearly completely.

We study the dynamics of relativistic electrons in the electromagnetic field obtained from PIC simulations. The test electrons are assumed to be injected near the cavity axis 

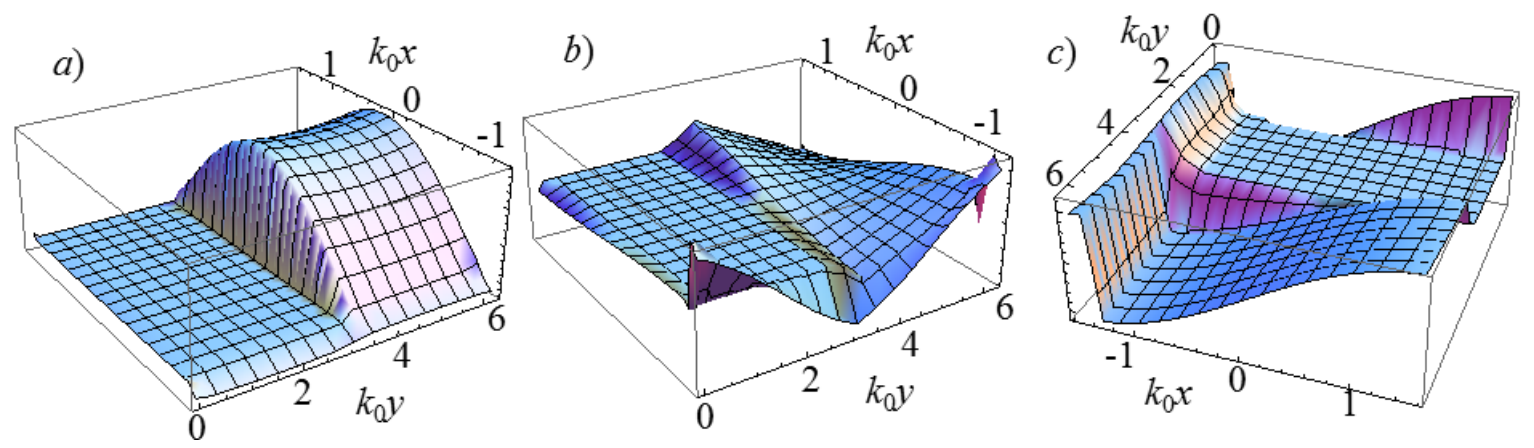

Figure 2: Snapshot of the fields (a) $E_{y}$ with the normalized magnitude $E_{y}=0.03$; (b) $E_{x}$ with the normalized magnitude $E_{x}=0.005$ and (c) $B_{z}$ with the normalized magnitude $B_{z}=0.005$. The fields are shown inside the cavity and in the damping part of the structure at the phase when the accelerating field $E_{y}$ reaches its maximum.

which passes through the cavity centers. First we estimate the gain in the momentum of the electron passing a one-period structure as a function of the initial field phase $\Theta$ and $x$-coordinate of the electron

$$
\begin{aligned}
\Delta p_{y} & \simeq \int_{0}^{T} e E_{y} d t, \\
\Delta p_{x} & \simeq \int_{0}^{T} e\left(E_{x}-B_{z}\right) d t,
\end{aligned}
$$

where $T=2 \pi / \omega_{L}$ is the laser period. The gain in the momentum of the electron travelling in the fields retrieved from PIC simulation as function of $\Theta$ and $x$ is shown in Fig.3. Electron acceleration is efficient when the gain in the longitudinal momentum peaks and the transverse momentum exhibits stable betatron oscillations. It follows from Fig $3 \mathrm{a}$ that the optimal regime of acceleration corresponds to $\Theta \approx 0$. In the ideal accelerator, the bunch axis must coincide with the middle of the microcavity where the transverse fields are zero. Numerical simulations show that the transverse force is not exactly absent at the cavity symmetry axis due to higher mode excitation and nonlinear interactions with plasma. As a result the electron undergoes some betatron oscillations even if it starts to move along the cavity symmetry axis.

Using periodic boundary condition for the field distribution shown in figure 2 we analyze electron acceleration in a many-period accelerating structure. The typical electron trajectories are shown in figure $3 \mathrm{~b}$. It follows from the analysis that the stable and efficient regime of electron acceleration corresponds to $\Theta \approx 0$ that agrees with the estimates presented above. 

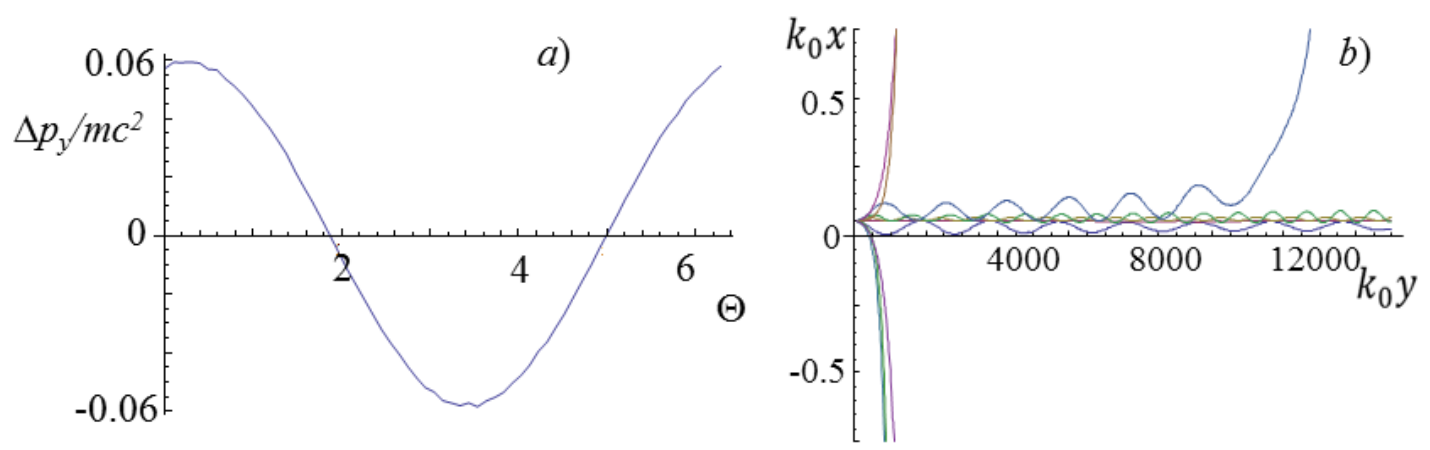

Figure 3: a) Gain in the logitudinal momentum $\Delta p_{y}$ of an electron passing through the one-period structure as function of the initial field phase $\Theta$; b) trajectories of electrons injected at the center of the cavity starting with $\gamma=200$, and evenly distributed phases in the field phase $\Theta$ between 0 and $2 \pi$.

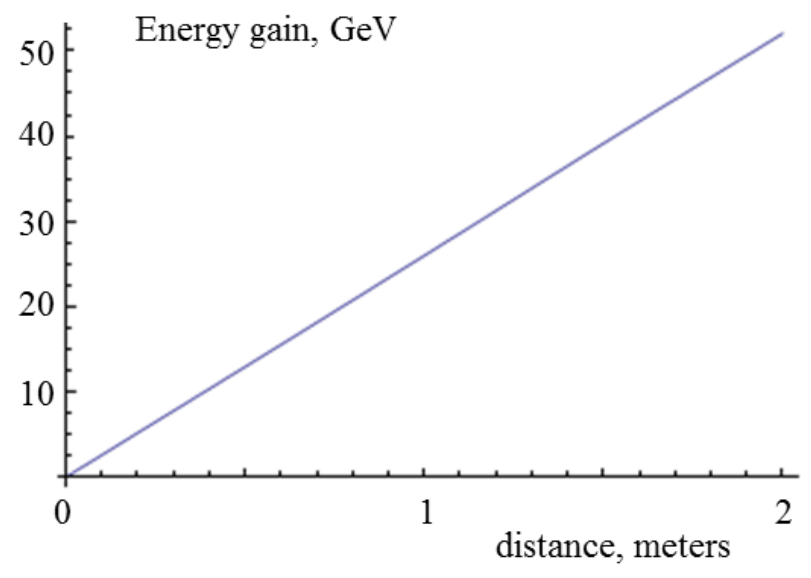

Figure 4: The dependence of electron energy on the passing distance. The starting parameters of the electron are $\gamma=200, x=0$ and $\Theta=-0.1 \pi$.

Long-term acceleration is demonstrated in Fig.4. The electron gains energy of about $50 \mathrm{GeV}$ over 2 meters of the structure. The transverse betatron amplitude of the accelerated electron remains less than $0.02 \lambda$ during acceleration.

\section{ELECTRON ACCELERATION IN OPEN PLASMA STRUCTURES}

The resonator microcavities have the advantage that they can trap the laser pulse energy and amplify the field. However, it might be difficult to achieve high finesses, and the resonat- 

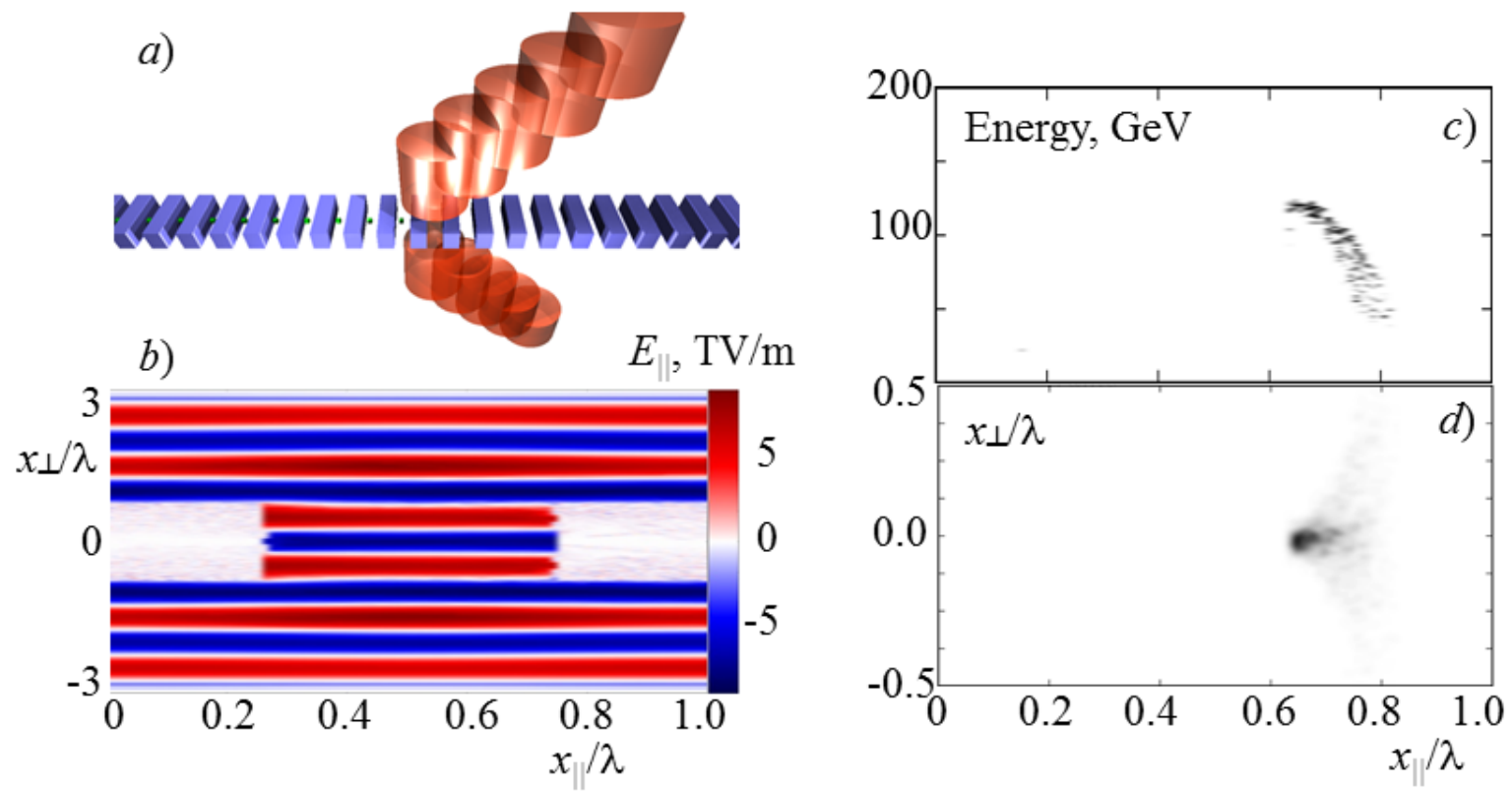

Figure 5: a) Three-dimensional view of the accelerating structure: two counterpropagating laser echelons hit a periodic plasma grid. b) The accelerating field component. c) Longitudinal electron phase space after the accelerating distance of $5 \mathrm{~cm}$. Electrons gained up to $120 \mathrm{GeV}$. d) Configuration space of the electron bunch. The coma of the bunch where the highest energy electrons are located has r.m.s. radius of about $20 \mathrm{~nm}$.

ing properties are very sensitive to the plasma parameters, sizes of the microboxes and the wall quality. In addition, the very thin semi-transparent foil covering the microcavity can only survive very short pulses of moderate intensity. Thus, non-resonating open structures have their advantages.

Again, to satisfy a phase synchronism between a normally incident laser pulse and an electron propagating in the transverse direction, the plasma structure must have a spatial period defined by the relation (3). Thus, for a relativistic electron, we need a structure with the period equal to the laser wavelength $\lambda$. One may think of different configurations. The simplest one is shown in Fig. 5 a. In this simulation we assume two counterpropagating phased laser echelons interacting with a periodic plasma grid. The overdense plasma regions have the length of $\lambda / 2$ and are separated by vacuum regions of the same length $\lambda / 2$. The accelerating field configuration is shown in Fig 5 b. We assumed that the lasers of the both echelons have the peak relativistic amplitude of $e E_{L} / m c \omega=1$ so that the combined accelerating field in the center of the structure reaches the peak amplitude $E_{\|}=2 E_{L}=7.5 \mathrm{TV} / \mathrm{m}$. 

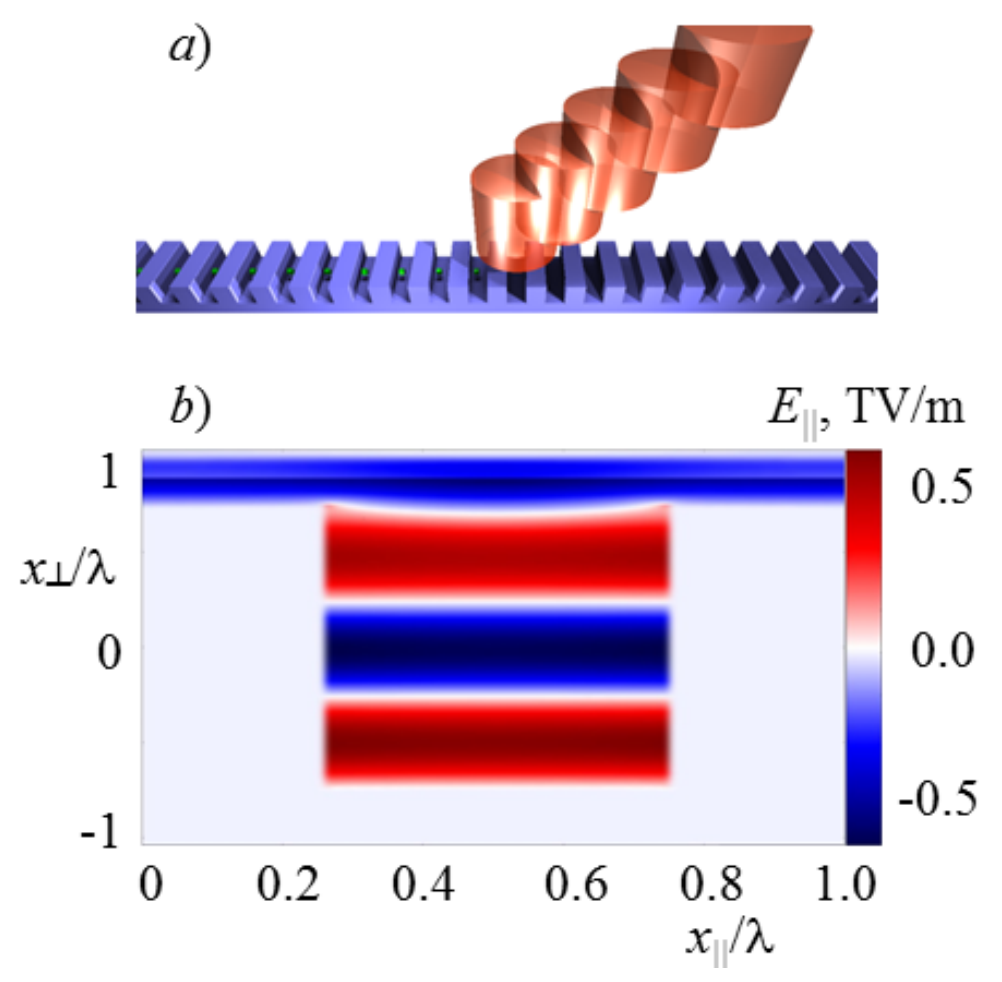

Figure 6: a) Three-dimensional view of the accelerating structure: a single laser echelon hits a periodic plasma grid on top of a plane substrate. b) The accelerating field component.

In our simulations, we injected a continuous beam of test electrons with initial $\gamma=200$ into the structure. About a quarter of the electrons has been trapped and accelerated up to $120 \mathrm{GeV}$ energy after a propagation distance of only $5.3 \mathrm{~cm}$, see Fig.5 5 . This corresponds to a sustained acceleration rate of $2.3 \mathrm{TeV} / \mathrm{m}$. The configuration space of the electron bunch is shown in Fig 5 d. Electrons with the highest energy are also tightly focused and the coma of the bunch has an r.m.s. radius of about $\sigma_{\text {r.m.s. }} \approx 20 \mathrm{~nm}$.

The advantage of the open structure with laser echelons coming from both sides is its robustness with respect to an exact position of the plasma grid. The fields are defined by the laser relative phases only. This makes the scheme unsensitive to e.g., mechanical jitter of the grid.

We mention also another importat feature. The plasma plasma structure remains invisible by the counterporpagating laser pulses. No modes modes propagating along the plasma grid are generated and we see essentially no perturbations in the laser field distribution in vacuum. This promises a high quality acceleration with potentially high energy conversion efficiency. 
Yet, a very similar field distribution can be created with a single echelon of laser pulses if we use a grid on a surface of a solid substrate, see Fig 6 a. The accelerating field inside the cavities, Fig.6b, has a very similar structure as in Fig.5p. In this particular simulation we used a lower laser pulse amplitude of of $e E_{L} / m c \omega=0.1$ so that the combined accelerating field of the incident and reflected wave in the center of the cavity reaches the peak amplitude $E_{\|} \approx 2 E_{L}=0.7 \mathrm{TV} / \mathrm{m}$. Because of the lower amplitude, the plasma response is more linear and less noise due to excitation of nonlinear harmonics and higher modes is seen in this picture. However, the exact position of the field maximum in this scheme is defined no more by the laser phase, but rather by the position of the reflecting surface of the substrate and thus is fully sensitive to mechanical oscillations of the structure.

\section{DISCUSSION AND CONCLUSION}

We have considered a possibility to use an echelon of laser pulses generated by the emerging CAN technology for direct particle acceleration in periodic plasma structures. The advantage of the method is the potentially extremely high acceleration rate above $\mathrm{TeV} / \mathrm{m}$. The reason is that the laser field itself is used and not a perturbative plasma field (e.g. wake field). In our simulations, we show that a sustained acceleration rate of $2.3 \mathrm{TeV} / \mathrm{m}$ is feasible with the plasma structures. We discussed resonant and free streaming configurations. The resonant plasma structures can trap energy of longer laser pulses that would be harvested by the witness bunch. However, the resonant structure is limited to moderate laser intensities

of about $10^{14} \mathrm{~W} / \mathrm{cm}^{2}$ as the thin semi-transparent plasma foil is destroyed very fast. The free streaming configurations can survive laser intensities above $10^{18} \mathrm{~W} / \mathrm{cm}^{2}$ for several tens of femtoseconds. The plasma structures survive a single shot only. However, due to their simplicity and projected very low production costs, the structures can be replaced for every laser shot at a kiloherz repetition rate.

\section{Acknowledgments}

We acknowledge very enlightening discussions with Prof. G. Mourou and Prof. T. Tajima.

This work has been supported by the Government of the Russian Federation (Project No. 14.B25.31.0008), by the Ministry of Science and Education of the Russian Federation, 
the Russian Federal Program "Scientific and scientific-pedagogical personnel of innovative Russia" (Agreement N8835)

[1] D. Strickland \& G. Mourou, Opt. Commun. 56, 219 (1985).

[2] S. P. D. Mangles, C. D. Murphy, Z. Najmudin, A. G. R. Thomas, J. L. Collier, A. E. Dangor, E. J. Divall, P. S. Foster, J. G. Gallacher, C. J. Hooker, Nature (London) 431, 535 (2004).

[3] C.G.R. Geddes, C. Toth, J. van Tilborg, E. Esarey, C.B. Schroeder, D. Bruhwiler, C. Nieter, J. Cary, and W.P. Leemans, Nature (London) 431, 538 (2004).

[4] J. Faure, Y. Glinec, A. Pukhov, S. Kiselev, S. Gordienko, E. Lefebvre, J.-P. Rousseau, F. Burgy, and V. Malka, Nature (London) 431, 541 (2004).

[5] W. P. Leemans, B. Nagler, A. J. Gonsalves, C. Tih, K. Nakamura, C. G. R. Geddes, E. Esarey, C. B. Schroeder, and S. M. Hooker, Nat. Phys. 2, 696 (2006).

[6] X. Wang, R. Zgadzaj, N. Fazel, Z. Li, S. A. Yi, X. Zhang,W. Henderson, Y.-Y. Chang, R.. Korzekwa, H.-E. Tsai, C.-H. Pai, H. Quevedo, G. Dyer, E. Gaul, M. Martinez, A. C. Bernstein, T. Borger. M. Spinks, M. Donovan, V. Khudik, G. Shvets, T. Ditmire \& M. C. Downer, Nature Comm. 4, 1988 (2013).

[7] A. Pukhov, J. Meyer-ter-Vehn, Applied Physics B 74 (4-5), 355-361 (2002).

[8] Y. Jeong, J. K. Sahu, D. N. Payne \& J. Nilsson, Electron. Lett. 40, 470 (2004).

[9] A. Liem et al., Conference on Lasers and Electro-Optics 2, 1067 (2004).

[10] T. Eidam et al., Opt. Express 19, 255 (2011).

[11] J. Prawiharjo et al., Opt. Express 16, 15074 (2008).

[12] G. Mourou, B. Brocklesby, T. Tajima and J. Limpert, Nature Photonics. 7, 258 (2013).

[13] C. Bellanger et al., Opt. Lett. 35, 3931 (2010).

[14] J. Rosenzweig, A. Murokh, and C. Pellegrini, Phys. Rev. Lett. 74, 2467 (1995).

[15] X. Eddie Lin, Phys. Rev. ST Accel. Beams 4, 051301 (2001).

[16] Y. C. Huang, D. Zheng, W. M. Tulloch, and R. L. Byer, Appl. Phys. Lett. 68, 753 (1996).

[17] R. B. Yoder and J. B. Rosenzweig, Phys. Rev. ST Accel. Beams 8, 111301 (2005).

[18] A.-C. Tien, et al., Phys. Rev. Lett. 82, 3883 (1999).

[19] T. Plettner, P. P. Lu, and R. L. Byer, Phys. Rev. ST Accel. Beams 9111301 (2006).

[20] A. Pukhov Journal of plasma physics 61, 425-433 (1999) 
[21] B. Shen and M. Y. Yu, Phys. Rev. Lett. 89 275004, (2002). 